\title{
Natural systems in building integrated aquaculture design
}

\author{
D. Damery, J. Webb, A. Danylchuk \& S. Hoque \\ University of Massachusetts Amherst, Dept. of Environmental \\ Conservation, Amherst, USA
}

\begin{abstract}
Aquaculture as a source of fish protein will continue to rise in importance in the coming decades. There exists the potential to develop Building Integrated Aquaculture (BIAq) incorporating holistic design approaches that will efficiently couple closed-loop aquaculture systems with the built environment. These integrated systems will become necessary if we are to develop local sources of food supply close to urban markets in temperate climates. This paper analyzes the role of nature in the design of such systems. We draw on the principles of Ecological Design to describe the role that natural systems can play in optimizing the performance of BIAq systems. We also incorporate a systems thinking approach in the context of BIAq design. A review of the literature analyzes the interaction of technical and natural systems in BIAq where the specific considerations for aquaculture production must be considered in concert with sustainable design of the built environment. Three main design issues will be explored: 1) Energy efficiency and the interaction of water mass with passive solar design including consideration of building envelope materials, space heating, water heating, and lighting demands; 2) Humidity control and natural ventilation; and 3) Natural hydroponic systems to close the nutrient loop with the aquaculture production systems. Design strategies are described and recommendations are provided.

Keywords: aquaculture, building integrated aquaculture, passive solar, natural systems, energy efficiency, humidity, natural ventilation, waste recycling.
\end{abstract}




\section{Introduction}

Fish is a lean, healthy alternative to other protein sources. Demand for fish is continually increasing due to global population growth and rising popularity among the developed world's consumers. Meanwhile, wild fisheries production began plateauing in the early 1990 s due to a combination of overfishing and environmental degradation. In the intervening 20 years, the rapidly emerging enterprise of aquaculture is attempting to meet the increased demand for fish. While exploding globally, regulations and economic barriers have thwarted commercial scale aquaculture development in the USA. As a result, we now import upwards of $83 \%$ of all seafood with almost half of this originating in overseas aquaculture [1].

This extreme reliance on imported aquaculture products faces criticism on two fronts. First, aquaculture processes are largely unregulated and are often socially, economically, and environmentally exploitive. Because awareness of the recent rise in aquaculture is low, many consumers are not cognizant that their fish are raised in unsustainable farms. Second, the long distance transport of seafood commodities is increasingly tied to oil prices and politics. Coupling the potential volatility in transport with the fact that Americans are only consuming approximately $3 / 8$ of the US Dept. of Agriculture-Health and Human Services recommended levels of this commodity, the extreme reliance on imports is alarming [2].

Given the high failure rate of large scale industrial aquaculture in the USA, there is increasing interest in small scale, local aquaculture enterprise. Local aquaculture also aligns with the growing consumer demand for locally produced food products, a phenomenon also reflected in the growth of communitysupported agriculture. While this can eliminate reliance on transport, the seasonal climate of many regions in the U.S. presents a significant hurdle to aquaculture. Fish are exothermic and require constant year round temperatures for growth, health, and survival. As a result, in much of the USA, winter may be too cool for 'warm-water' species such as tilapia, summer may be too warm for 'cool-water' species such as trout. For successful small scale aquaculture in northern latitudes, producers will have to address the issue of temperature control through innovative system design and engineering as a way to make local aquaculture economically viable.

In the past, aquaculture and building design were generally considered separately. Given the potential synergies in these disparate disciplines, adopting a more holistic concept of Building-Integrated Aquaculture (BIAq) is now being explored. Specifically, this paper assesses the role natural systems can play in optimizing BIAq performance.

\section{BIAq systems design}

As McHarg [3] points out, an ecological view includes the notion that the world and evolution are part of a creative process, and similarly, the design of BIAq systems can be viewed as a work in process. This is a new field and much work 
remains to be done. In this paper, we will illuminate aspects where the science and technology of BIAq systems can be advanced through careful design that incorporates natural processes.

\subsection{Systems thinking}

The depletion of natural fish stocks is interconnected with the failure to incorporate systems thinking in understanding the dynamics of fisheries ecosystems [4]. Furthermore, the complex nature of fisheries management and the global collapse of natural fisheries have been impacted by the availability of cheap fossil fuels. These facts point to the opportunity, and growing need, for aquaculture production systems specifically designed for sustainability.

Traditional, pond or pen based, aquaculture systems are examples of open systems. BIAq systems, in contrast, incorporate Recirculating Aquaculture Systems (RAS) that are closed loop systems. McHarg's [3] analysis of a closed system for human space transport has many parallels with the design considerations of BIAq systems. The space transport system consisted of three related systems including a source of power (the sun, or a fluorescent lamp) which supported an oxygen-carbon dioxide cycle with algae, supported by the solar power giving off oxygen (consumed by the human) and consuming carbondioxide (waste) given off by the human. The second system was the water system, with the human consuming some water, giving off waste (urine) that was consumed by the algae, which in turn purified and produced clean water. The final system described was the food system, with the human consuming algae, giving off waste (excrement) that was used, in turn, as a feed input for the algae. In this example it is clear that natural processes, specifically algal growth would be critical for a sustainable long-term life-support environment for space travel. We can apply similar systems thinking in BIAq to move aquaculture production closer to the goals of sustainability.

To extend McHarg's model to BIAq design, a detailed understanding of water's role as the conduit of mass and energy flows (dissolved gases, waste metabolites, heat, etc.) in an aquaculture system creates opportunities for better integration of systems and buildings. Well thought out compartmentalization of specialized active and passive control systems coupled with efficient recirculation of water is key to BIAq design. Designers should incorporate passive solar technologies to increase the proportion of total system heating and energy demand that is provided directly from this renewable source. Specifically, Hoque et al. [5] call for the use of renewable energy and passive solar systems to assist in regulating water temperature, controlling indoor air quality, and reducing other energy loads such as lighting. They propose solar heated reserve water heat exchangers as an efficient mechanism for RAS water temperature control.

\subsection{Water tank geometry}

Water is essential to any aquaculture enterprise. Because indoor space is relatively expensive in comparison to outdoor pond systems, BIAq facilities 
require intensive recirculating aquaculture systems (RAS). RAS size is frequently gauged by total water volume with most designs devoting upwards of $80 \%$ of the total system volume to fish culture. Best management practices suggest water temperatures should remain relatively constant. How to best apportion this water within an indoor space is not clearly defined. Several issues arise: tank shape, space utilization, and accessibility; the ratio of total system water volume-to-building air volume; and the management of water/air interfaces.

In the case of natural wild fisheries, production occurs in lakes and seas that tend to have a large surface area to volume ratio (i.e. they are wide and shallow). This geometry is inefficient from the perspectives of energy and material usage in traditional building construction that would favor a cube, or even a sphere if un-constrained by traditional materials and building practices. Furthermore, as discussed below, operational considerations seem to favor configurations that might include multiple cylindrical tanks.

From a volume utilization standpoint, square or rectangular tanks extending from floor-to-ceiling use indoor space most efficiently. Unfortunately, such designs are generally impractical when it comes to the culture of finfish species. In cylindrical tanks, hydrodynamics can be adjusted to create a 'self-cleaning' process that significantly improves water flow. This process is difficult to achieve in rectangular tanks, and consequently, fish density and health are compromised. Deep (or tall) tanks also present problems. Many finfish species do not fully utilize the water column and prefer either the surface or bottom of the tank. Tanks extending to the ceiling present a number of management considerations related to access and occupational hazards. Building designers must work together with aquaculture process engineers to not only ensure fish can be cultured, but also sampled, graded, transferred, and harvested.

From an aquaculture standpoint, cylindrical tanks with a 3:1 to 5:1 diameter to height ratio are preferred. Fitting tanks of these dimensions into a rectangular building presents several issues. Systems employing small tanks result in a significantly lower total growing volume than do systems using large tanks. Utilization of floor space is not the only concern as the ratio of total system water volume-to-building air volume is an important consideration in the design of the facility's heating/cooling systems. BIAq enterprise requires a range of tank sizes (small tanks for larval fish, medium for fingerlings, and large for grow-out), the relationship between tank dimensions, size, floor space, and water-to-air ratio must be carefully considered. In the case of very large tanks, one must question whether buildings are even appropriate at all. One solution is for exceedingly large tanks to become buildings in themselves, sharing structural elements and providing cost savings over the construction of separate tank and building entities.

\subsection{Indoor air quality}

Natural fisheries are directly connected, and form an integral part of, global climate systems including the water cycle, carbon cycle, oxygen cycle and 
others. A BIAq enterprise must manage water quality for oxygen levels and nutrient loading.

An additional consideration of design is related to the management of airwater interfaces. Tank design contributes to this issue through its impact on total water surface area. Cylindrical tanks with $3: 1$ to 5:1 ratios have a considerably greater surface area than rectilinear tanks. Surface area not only contributes to heat loss, but also evaporation. While tank covers are a potential solution, aquaculture management issues must be considered. Aside from fish culture tanks, surface area must be managed in other parts of an aquaculture system. In fact, often overlooked is the requirement for surface exposure during the process of removing the high levels of dissolved carbon dioxide produced by fish from recirculated water. In this case, surface area and exposure to fresh air is generally increased through the use of counter current exchangers. Due to the potential toxicity of carbon dioxide, such devices must be vented to the environment, creating a significant pathway for heat loss and evaporation. A BIAq enterprise needs to consider the sometimes conflicting requirements for water surface exposure.

Given the open nature of wild fisheries in marine and freshwater ecosystems, air quality is not a consideration. Contact between pond, lake or sea surfaces with the atmosphere connects the aquatic systems with global cycles for water and other atmospheric components. BIAq design, however, requires mechanical systems to manage air quality to acceptable levels, but the energy costs of these systems have to be weighed. Hoque et al. [5] propose mechanical solutions to manage dehumidification and air exchange to provide adequate indoor environmental quality and minimize energy losses through ventilation.

Looking to nature for inspiration for the control and management of indoor environmental quality is at present an unexplored area of research. Mechanical systems are clearly required for the control of $\mathrm{CO}_{2}$ levels, relative humidity, and air temperature inside buildings. However, considering the linkages between these parameters and the requirements of integrated crops systems, further exploration into natural systems will likely yield beneficial insight in the future.

\subsection{Waste control}

McDonough and Braungart [6] promoted the notion of waste equals food. BIAq design using RAS technologies must find a productive natural process to manage the effluent waste from the fish production system.

Producing agricultural products from waste streams has a long history. For centuries before the advent of the Haber-Bosch process and inexpensive inorganic fertilizers, farmers employed natural manures from livestock to their crops. Such practices continue today, often under the label of 'organic production'. While pond aquaculture has long employed manures to stimulate primary production, only in the last 20 years or so have people realized the value of aquaculture wastewater in plant production. Through the 'village aquaculture' approach, Brummett and Costa-Pierce [7] present a fish pond as the centerpiece in a village also using the pond water for irrigation. Going a step further, Rakocy [8] found commercial levels of production in field grown bell peppers utilizing 
fish culture water from more intensive aquaculture tilapia production. Given that high fish density RAS employ devices with the potential to capture, concentrate, and move effluent to crop systems BIAq facilities present even greater opportunities for crop integration.

While Costa-Pierce and Desbonnet [9] claim that production of fish and crops utilizing wastewater has occurred throughout recorded time, their research focused on an ecosystem approach to managing urban wastewater raised additional health concerns. The direct application of BIAq effluent to food crops presents similar concerns. In response, alternative non-food natural systems have been employed such as marsh systems that utilize waste material in a reed bed system. Hutchinson [10] illustrated the practice of using reed beds to treat wastewater while also incorporating algae that was then consumed by Daphnia (water fleas) that were in turn used as feed for cultured finfish. An additional inspiration from nature is the utilization of aquaculture waste in silvofisheries; aquaculture operations are combined with tree growth for control of waste. Fitzgerald [11] describes a number of examples of brackish water aquaculture systems incorporating mangrove forests to utilize the fish waste. The relatively recent development of biofuels presents additional opportunities for integration with non-food crops such as sunflower, jatropha, and microalgae.

It is noteworthy that the majority of these examples involve warm water fisheries and warm outdoor climates. Consequently, it is uncertain whether this model can be utilized in temperate BIAq systems without overcoming significant technical hurdles. Due to space and sunlight requirements of the integrated plant systems, their incorporation favors production outside the building envelope containing the fish production tanks. This presents an additional design challenge of maintaining water temperature if the wastewater is circulated out of the building envelope and into an external environment. Equally difficult is maintaining the integrated plant systems within a thermally controlled greenhouse. While some have suggested that high density integrated hydroponics using efficient LED lighting within the building envelope is the answer, the economic feasibility of such designs has not been established.

\section{Conclusions and recommendations}

As Odum [4] argues we are currently in an era reliant on reserves of fuels that have been stored over geological time and need to move toward more renewable sources of power. BIAq and natural systems design can reduce our reliance on fossil fuels in two ways. First, by reducing fuel use during the transportation and delivery of fish protein from distant tropical production environments to urban temperate markets. Secondly, by optimizing building energy use and capitalizing on the synergies between aquaculture systems and their building envelope.

Odum [12] refers to the potential for human's reaction to periods of excess consumption by being adaptive. We believe that BIAq is an example of this. We also stress that new aquaculture systems must be designed using closed-loop systems as a guiding principle. New research into optimal tank/building configurations is needed. Future BIAq design will also require further 
consideration of tank geometry, operational processes, building energy, thermal control, humidity, and indoor air quality performance. It will necessitate optimizing the process of incorporating natural systems in waste control, such as agricultural production. And finally, as a commercial enterprise, sustainable aquaculture must also be economically viable. Consequently, the role product cost will play in the evolution of BIAq designs must not be overlooked.

\section{References}

[1] Van Voorhees, D. and Lowther, A. Fisheries of the United States 2009. National Oceanic and Atmospheric Administration annual report. Silver Springs, NOAA. p.104, 2010.

[2] USDA. Report of the dietary guidelines advisory committee on the dietary guidelines for Americans, Washington, DC. USDA-ARS/HHS. p.445, 2010.

[3] McHarg, I.L., Design with Nature, The Natural History Press, New York, pp.43-53,1969.

[4] Odum, H.T., Environment, Power, and Society, Columbia University Press, New York, p. 380, 2007.

[5] Hoque S., Webb, J., and Danylchuk, A. Building Integrated Aquaculture: can holistic designs increase system efficiencies and make recirculating aquaculture more successful in the northeastern United States? ASHRAE Journal. [Accepted for publication]

[6] McDonough, W. and Braungart, M. Cradle to Cradle, North Point Press, New York, 2002.

[7] Brummett, R. and Costa-Pierce, B. "Village-based aquaculture ecosystems as a model for sustainable aquaculture development in Sub-saharan aquaculture" in Ecological Aquaculture edited by B. Costa-Pierce, Blackwell Publishing, Oxford, UK. pp.145-160, 2002.

[8] Rakocy, J. "An Integrated Fish and Field Crop System for Arid Areas" in Ecological Aquaculture edited by B. Costa-Pierce, Blackwell Publishing Oxford, UK, pp.263-285, 2002.

[9] Costa-Pierce, B. and Desbonnet, A. "A Future Urban Ecosystem Incorporating Urban Aquaculture for Wastewater Treatment and Food Production" in Urban Aquaculture edited by B. Costa-Pierce, A. Desbonnet, P. Edwards and D. Baker, CABI Publishing, Wallingford, UK pp.1-14, 2005.

[10] Hutchinson, L. Ecological Aquaculture. Permanent Publications, East Meon, England, 2005. p.31.

[11] Fitzgerald, W. "Silvofisheries: Integrated Mangrove Forest Aquaculture Systems" in Ecological Aquaculture edited by B. Costa-Pierce, Blackwell Publishing Oxford, UK, pp.161-262, 2002.

[12] Odum, H.T. Systems Ecology, John Wiley and Sons, New York, 1983. 